\title{
0246-P1007 Insulin resistance in patients with thyroid dysfunction and hepatosteatosis
}

SO Keskek, S Kirim, R Kaya, D Tuzun, G Ortoglu, T Saler

Numune Education and Training Hospital, Department of Internal Medicine, Adana, Turkey

Introduction: Hepatosteatosis is one of the results of insulin resistance. Thyroid dysfunctions effect on insulin sensitivity. The aim of this study was to evaluate insulin resistance in patients with hepatosteatosis and either hypothyroidism or hyperthyroidism.

Design: A total of 407 patients with hepatosteatosis were included. These patients were further divided into two study subgroups and a control group: 102 subjects with hypothyroidism, 103 with hyperthyroidism and 202 with normal thyroid function in the control group. The institution review board of hospital approved the study. Serum TSH, free T4, free T3 concentrations, blood glucose and insulin levels, serum lipid levels, hepatic transaminases and homeostasis model assessment of insulin resistance (HOMA IR) were measured. Insulin resistance was calculated according to HOMA index and compared between the groups. IBM statistics 20.0 for Windows was used for statistical analysis. Ki square and ANOVA tests were used for comparing groups.

Results: Average age was 50.8 \pm 14.1 years. Male/female ratio was 141:266. Frequencies of insulin resistance in patients with hepatosteatotis and either hypothyroidism, hyperthyroidism, or normal thyroid function were $43 \%, 40 \%$ and $48 \%$ ( $P$, nonsignificant), respectively. HOMA-IR indices were not statistically different between groups $(P=0.104)$ (Table 1$)$.

Conclusions: Hypothyroidism and hyperthyroidism are not correlated to insulin resistance in patients with hepatosteatosis. We decided that patients with hepatosteatosis already have insulin resistance despite different associated comorbidities. Similar studies in literature did not emphasize on hepatosteatosis in such cases.

Keywords: Insulin resistance, hepatosteatosis, hypothyroidism, hyperthyroidism

\begin{tabular}{|lccccc|}
\hline Parameter & $\begin{array}{c}\text { Normal } \\
\text { Range }\end{array}$ & $\begin{array}{c}\text { Control } \\
(\mathrm{N}=202)\end{array}$ & $\begin{array}{c}\text { Hypothyroidism } \\
(\mathrm{N}=102)\end{array}$ & $\begin{array}{c}\text { Hyperthyroidism } \\
(\mathrm{N}=103)\end{array}$ & $P$ \\
\hline TSH (mcIU/ml) & $0,57-5,56$ & $2,97 \pm 1,1$ & $18,22 \pm 7,4$ & $0,16 \pm 0,08$ & 0,001 \\
$\begin{array}{l}\text { Insulin } \\
\text { resistance } \\
\text { frequency }\end{array}$ & - & $97(48 \%)$ & $43(43 \%)$ & $41(40 \%)$ & 0,497 \\
HOMA-IR & $<2,5$ & $3,5 \pm 3,7$ & $3,2 \pm 2,1$ & $3,3 \pm 3,1$ & 0,104 \\
\hline
\end{tabular}

Table 1: Comparison of groups according to the HOMA-IR and insulin resistance frequency. 